\title{
Cross-modal generalization to tone and light in human eyelid conditioning'
}

\author{
G. A. MARLATT, D. LILIE, B. D. SELVIDGE, M. D. SIPES AND I. GORMEZANO
}

INDIANA UNIVERSITY

Cross-modal generalization was examined with an experimental design permitting an assessment of non-associative factors. It was found that relative to Ss receiving the orthodox unimodal CS in extinction, cross-modal Ss demonstrated about $60 \%$ generalization. Furthermore, cross-modal CRs were significantly greater in frequency than those given by cross-modal non-associative control Ss given unpaired CS and IIS presentations in acquisition.

Pavlov (1927) reported the pervasive occurrence of cross-modal generalization (CMG), and Razran (1949) has described Russian conditioning experiments in which CRs generalized across sense modalities with strengths up to $38 \%$. Kimble $(1961$, p. 361$)$ while expressing some doubt as to the purported magnitude of CMG, has suggested that it bears importantly on the "failure-ofdiscrimination" interpretation of primary stimulus generalization. Thompson (1959) has indicated that the control group in sensory preconditioning experiments have simply tested for CMG, and that typically little or no such generalization is found. Yet, Thompson presented evidence of CMG, and found that its magnitude decreased with increased acquisition training. Thompson employed an orthodox unimodal control group for assessing CMG, but as yet, no study has attempted to control for the possible contribution of sensitization or pseudo-conditioning. The present investigation bears on this question.

Method

The apparatus was essentially the same as that described by Moore \& Gormezano (1961). In brief, Ss' room contained two identical enclosures which permitted two Ss to be run simultaneously. The Ss wore elastic headbands supporting a stimulus air jet and a rotary potentiometer for recording eyelid movement from the stimulated (right) eye. An abrupt change in hue (to pale orange) and an increase of illumination (from 5.6 to 6.7 apparent $\mathrm{ft}-\mathrm{cd}$ ) of a $10-\mathrm{cm}$ milk-white glass disk served as the visual CS. A speaker, flush with the compartment ceiling, delivered a tone CS psychophysically equated in "intensity" by the method of adjustment to the visual CS, and consisted of a 1100-cps tone of $51 \mathrm{~dB}$ SPL. The duration of both CSs was $600 \mathrm{msec}$. and the CS-US interval $500 \mathrm{msec}$. A 100-msec. puff of compressed nitrogen served as the US, and was of sufficient intensity at the eye to support a $120-\mathrm{mm}$ column of mercury.

The Ss were 49 men and 79 women from introductory psychology classes at Indiana University. Sixteen Ss were randomly assigned in pairs to the eight cells of a factorial design in which the dimensions were: (1) Paired vs. Unpaired CS and US presentations; (2) Crossmodal (CM) vs. Unimodal (UM) CS presentations (a dummy variable in acquisition); and (3) Auditory vs. Visual CS. The Ss were given "neutral" instructions virtually identical to those of Gormezano \& Moore (1962). The instructions differed in sub-groups only in terms of the CS stimulus Ss would receive in acquisition. The four Paired groups received 70 presentations of the relevant CS during acquisition, $56(80 \%)$ of which were reinforced, at randomized intertrial intervals of 25,30 , and $35 \mathrm{sec}$. with a mean of $30 \mathrm{sec}$. The four Unpaired groups received 70 presentations of the relevant CS alone and 56 presentations of the US alone, with the restriction that no more than five CS-alone nor four US-alone trials occur successfully. In the Unpaired groups the intertrial intervals in acquisition were randomized at 10,15 , and $25 \mathrm{sec}$. with a mean of $16.6 \mathrm{sec}$. In extinction, all groups received 20 presentations of the relevant $\mathrm{CS}$ alone, at an average intertrial interval of $30 \mathrm{sec}$. A 1:1 ratio of amplification prevailed between lid movements and deflections of the recording pen. In acquisition, any deflection from baseline of at least $1-\mathrm{mm}$ in the 150-550 msec. interval after CS onset were scored as CRs. This interval was extended to $600 \mathrm{msec}$. in extinction.

\section{Results}

Figure 1 presents the performance curves of the Paired-Unpaired and CM-UM dimensions with the ToneLight dimension collapsed. Examination of the figure reveals that under the Paired CM and UM conditions

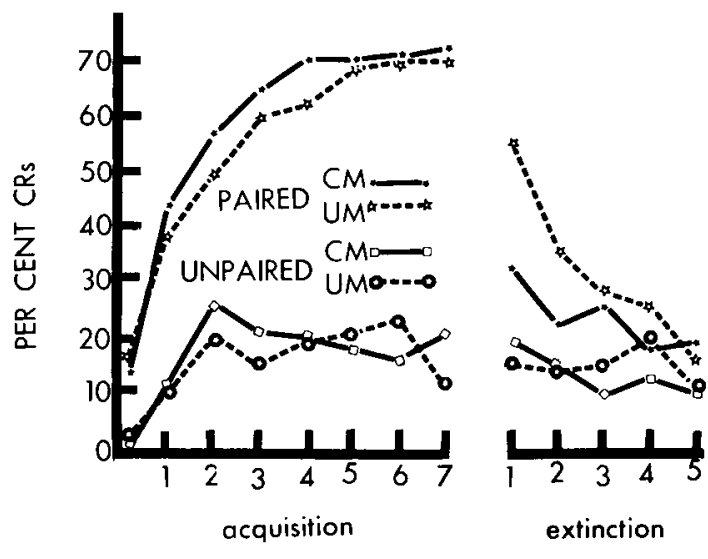

BLOCKS OF TRIALS

Fig. 1. The percentage of responses plotted in 10-trial blocks during acquisition and 4-trial blocks during extinction. 
asymptotic levels of about $70 \%$ were attained in acquisition, while for the Unpaired CM and UM conditions the response level was about $20 \%$. In extinction the Paired CM condition revealed substantially fewer responses than the Paired UM condition, and indicated the decremental effects on response frequency of the crossmodal shift in the CS. The response frequency under the Paired $\mathrm{CM}$ condition, however, was greater than under the Unpaired CM condition. An analysis of variance on the arc-sine transform of individual percentage scores on the 70 acquisition and 20 extinction trials revealed a significant effect for the Paired-Unpaired dimension in acquisition ( $F=17.39 ; \mathrm{df}=1 / 120 ; \mathrm{p}<.01)$ and extinction $(F=7.87 ; \mathrm{df}=1 / 120 ; p<.01)$. The CM-UM and Tone-Light main effects and all interaction effects failed to attain significance in acquisition or extinction (all $p^{\prime} s>.05$ ). To test for CMG a t-test was carried out on the mean percentage responses for the Paired vs. Unpaired CM Groups on the first 4-trial block of extinction. The frequency of CRs given under the Paired $C M$ condition was significantly greater $(t=2.16$; $d f=$ $1 / 62 ; \mathrm{p}<.05)$, indicating that the $C M G$ effect was not due to sensitization or pseudoconditioning, but to prior associative learning. A t-test was also performed on the mean percentage of responses of the Paired CM vs. UM conditions on the first extinction block. The results revealed a significantly lower level of responding for the Paired CM condition $(t=2.13 ; \mathrm{df}=1 / 62 ; \mathrm{p}<.05)$, indicating that the cross-modal shift in the CS had a reliable decremental effect on response frequency. Discussion

The major finding of the presentinvestigation was the observation of a CMG effect that could notbe attributed to the non-associative factors of sensitization or pseudoconditioning. Furthermore; compared with the orthodox UM conditioning, Paired CM Ss showed about $60 \%$ generalized responses during the first four-trialblock of extinction. This amount of generalization compares favorably with eyelid conditioning studies which have tested for stimulus generalization within the same same sense modality. Thus, Vandament \& Price (1964) employing two intensities of a light CS, and taking the first trial of extinction as the test for generalization, reported a drop from $90 \%$ to $70 \%$ CRs under acquisition training of $100 \%$ reinforcement, and a drop from $70 \%$ to $50 \%$ when prior reinforcement was held at $50 \%$.

Kimble (1961) has suggested that the phenomenon of CMG precludes the interpretation of primary stimulus generalization as a "failure-of-discrimination" because the confusion of stimuli, as in the present investigation, seems improbable. Prokasy \& Hall (1963) have suggested, however, that " . . . changes in physical events which necessarily occur with successive event presentation may not, so far as the subject is concerned, constitute a change at all" (p.316). That is, in the present investigation, although the light and tone CS were clearly discriminable, S's perception of the signalizing role of the CS may have constituted the basis of equivalence. However, such an explanation can not account for the greater decrease in response frequency under the $\mathrm{CM}$ condition relative to the orthodox UM condition. But Prokasy \& Hall (1963) further suggest that a change in the physical characteristics of the CS may induce orienting (or alpha) responses which can act to suppress CRs. Some modest support for this contention was in fact found in the present data. Examination of the frequency of alpha responses following the cross-modal shift revealed a substantial increase in the frequency of alpha responses given by the Paired CM groups, whereas no such increment was noted for either the Unpaired CM or Paired UM groups. Although the differences did not attain statistical significance $(p>.05)$ the implications would be considerable if it were later shown to be a reliable outcome.

\section{References}

Gormezano, I., \& Moore, J. W. Effects of instructional set and UCS intensity on the latency, percentage, and form of the eyelid response. J. exp. Psychol., 1962, 63, 487-494.

Kimble, G. A. Hilgard and Marquis' conditioning and learning. New York: Appleton-Century-Crofts, 1961.

Moore, J. W., \& Gomezano, I. Yoked comparisons of instrumental and classical eyelid conditioning. J. exp. Psychol., 1961, 62, $552-559$.

Pavlov, I. P. Conditioned reflexes. London: Oxford University Press, 1927. (Transl. by G. V. Anrep.)

Prokasy, W. F., \& Hall, J. F. Primary stimulus generalization. Psychol. Rev., 1963, 70, 310-322.

Razran, G. Stimulus generalization of conditioned responses. Psychol. Bull., 1949, 46, 337-365.

Thompson, R. F. Effect of acquisition level upon the magnitude of stimulus generalization across sensory modality. $J$. comp. physiol. Psychol., 1959, 52, 183-185.

Vandament, W. E., \& Price, L. E. Primary stimulus generalization under different percentages of reinforcement in eyelid conditioning. J. exp. Psychol., 1964, 67, 162-167.

\section{Nofe}

1. This research was supported by Grant GB-2843 from the National Science Foundation. 